\title{
Antiretroviral Therapy in HIV/HCV Co-Infection Italian Consensus Workshop
}

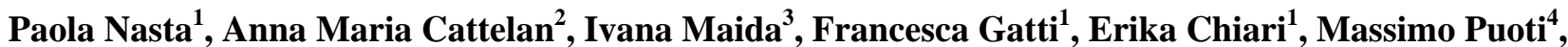 \\ Giampiero Carosi ${ }^{1}$ \\ ${ }^{1}$ Clinic of Infectious and Tropical Diseases, Spedali Civili Hospital, Brescia, Italy; ${ }^{2}$ Clinic of Infectious Diseases, Hospital of Rovigo, \\ Rovigo, Italy; ${ }^{3}$ Institute of Infectious Disease, University of Sassari, Sassari, Italy; ${ }^{4}$ Infectious Diseases Department, Azienda Osped- \\ aliera-Ospedale Niguarda Cà Granda, Milan, Italy. \\ Email:paola.nasta@tin.it
}

Received March $18^{\text {th }}, 2013$; revised April 26 $6^{\text {th }}, 2013$; accepted May $26^{\text {th }}, 2013$

Copyright (C) 2013 Paola Nasta et al. This is an open access article distributed under the Creative Commons Attribution License, which permits unrestricted use, distribution, and reproduction in any medium, provided the original work is properly cited.

\begin{abstract}
About $50 \%$ of people living with the HIV infection in Italy are co-infected with $\mathrm{HCV}$. In this group of patients, the primary cause of mortality is liver disease, which accounts for up to $14 \%$ of deaths. HIV/HCV co-infection also exposes patients to a higher risk of progression to AIDS, a faster evolution towards cirrhosis, more frequent drug toxicity, and lower tolerance for antiretroviral therapy. Moreover, HCV infection can play a part in increasing immune system depression; neurological, cognitive and renal damage; and bone fragility. Hence an optimal antiretroviral regimen needs to be chosen for co-administration with anti-HCV therapy and timed appropriately to improve the prognosis of co-infected $\mathrm{HIV} / \mathrm{HCV}$ patients. Unfortunately, however, data on the safety and efficacy of antiretroviral drugs in these patients is scarce, as are studies of pharmacokinetics in patients with advanced liver impairment. Furthermore, restoring adequate immune constitution seems not to slow the progression of liver disease, and the metabolic and hepatic toxicity of some antiretroviral drugs can even contribute to inflammatory and fibrogenic processes. It is therefore essential that HIV/ $\mathrm{HCV}$ co-infected patients receive only medications capable of ensuring the best immune recovery but possessing the lowest potential to trigger immune reconstitution syndrome or hepatic and metabolic damage.
\end{abstract}

Keywords: ART; HIV/HCV; Co-Infection; Liver Toxicity

\section{Consensus Development}

The consensus meeting, held under the auspices of the Italian Society for Infectious and Tropical Diseases (Società Italiana di Malattie Infettive e Tropicali-SIMIT) and the Italian Society for Sexually Transmitted Diseases (Società Italiana di Malattie Sessualmente TrasmessibiliSIMAST), and the patronage of the Italian Infectious Disease and Public Health Foundation (Fondazione di Malattie Infettive e Salute Internazionale-MISI) was funded by an unrestricted grant from ViiV Health Care, Italy.

The first draft of "Antiretroviral Therapy in HIV/HCV Co-infection, Italian Consensus Workshop" was written by Dr. Paola Nasta, Dr. Anna Maria Cattelan, Dr. Ivana Maida and Dr Francesca Gatti with the help of Erika Chiari. The draft was reviewed by Prof. Raffaele Bruno (IRCCS San Matteo Hospital, University of Pavia, Division of Infectious and Tropical Diseases, Pavia, Italy), Prof. Roberto Cauda (Catholic University of the Sacred
Heart, Institute of Clinical Infectious Diseases, Rome, Italy), Dr. Simona Di Gianbenedetto (Catholic University of the Sacred Heart, Institute of Clinical Infectious Diseases, Rome, Italy), Prof. Giovanbattista Gaeta (Department of Clinical and Experimental Medicine and Surgery "F. Magrassi-A. Lanzara", Second University of Naples, Italy), Dr. Francesco Mazzotta (Santa Maria Annunziata Hospital, Infectious Diseases Department, Florence, Italy), Prof. Evangelista Sagnelli (Department of Public Medicine, Section of Infectious Diseases, Second University of Naples, Italy), Prof. Gloria Taliani (La Sapienza University, Institute of Infectious and Tropical Diseases, Rome, Italy) and Dr. Carlo Torti (University of Brescia, Institute of Infectious and Tropical Diseases, Brescia, Italy), and revised accordingly by Prof. Giampiero Carosi and Prof. Massimo Puoti.

The following doctors participated in the work group: Marino Bonasso, Vanni Borghi, Federica Borghi, Silvana Caprioglio, Erika Chiari, Anna Maria Degli Antoni, 
Palma Delle Foglie, Eugenia Di Meco, Gian Piero D'Offizi, Concetta Donato, Francesco Esperti, Roberto Gulminetti, Giancarlo Iaiani, Nicoletta Ladisa, Davide Motta, Myrvete Muca Perja, Giorgio Perboni, Emanuele Pontali, Vincenzo Portelli, Alessandro Ruggeri, Vincenzo Sangiovanni, Paolo Scerbo, Vincenzo Selva, Filomena Simeone, Hajrijie Skiti and Gabriella Verucchi.

\section{Introduction}

Human Immunodeficiency Virus (HIV) and Hepatitis C Virus (HCV) share common transmission pathways, although $\mathrm{HCV}$ is more efficiently transmitted through blood than the sexual route. It is unsurprising therefore that roughly half of the Italian population of HIV patients also test positive for $\mathrm{HCV}$. In fact, $\mathrm{HCV}$ is the most prevalent cause of liver disease in co-infected Persons Living with HIV (PLWHIV), which is in turn the most common cause of non-AIDS-related mortality in these patients, accounting for up to $14 \%$ of deaths worldwide. In addition, HCV co-infection worsens immune depression and contributes to neurological, cognitive and renal impairment, as well as bone fragility. It may also expose patients to a higher risk of progression to AIDS, to more frequent drug toxicity, and to decreased tolerance for antiretroviral therapy $[1,2]$.

Nevertheless, combined antiretroviral therapy (cART) has been linked to a reduction in liver-related mortality in PLWHIV, acting via suppression of HIV replication and restoration of CD4 counts. That being said, data on the safety and efficacy of antiretroviral drugs in these subjects are still scarce, as are pharmacokinetic/pharmacodynamic studies in patients with advanced liver disease. It seems, however, that the timing of cART initiation and the choice of anti-HIV medications are critical for the prognosis of PLWHIV.

Under the auspices of the Italian Society for Infectious and Tropical Diseases (Società Italiana di Malattie Infettive e Tropicali-SIMIT), and Italian Society for Sexually Transmitted Diseases (Società Italiana di Malattie Sessualmente Trasmissibil-SIMAST), a group of experts in the field of ART was invited to discuss the current risks and benefits of antiretroviral treatment in $\mathrm{HIV} / \mathrm{HCV}$ co-infected patients, and to update the statements published following the first Italian Consensus Conference in 2005.

The experts prepared a draft of the consensus statements after a careful review of the current literature on three key issues of optimized antiretroviral treatment in HIV/HCV co-infected patients:

1) Management of HIV/HCV in co-infected patients.

2) Optimization of cART in HIV/HCV co-infected patients who are not eligible for pegylated-interferon (PegIFN) and ribavirin (RBV) treatment, or those who are awaiting new anti-HCV treatment options.
3) Optimization of cART in co-infected patients suitable for anti-HCV treatment with the current peg-IFN/ $\mathrm{RBV}$ regimen.

Statements were graded for their strength and evidence based on the criteria adopted by the Infectious Diseases Society of America (IDSA) (Table 1), and scrutinized and discussed by a qualified group of Italian infectious disease specialists, hepatologists, and virologists, convened in Sirmione for a 2-day workshop. After a series of introductory lectures, the previously prepared statements were analysed and revised by the specialist physicians in work groups of $5-10$. On the second day of the consensus meeting, the revised statements were presented in a plenary session by the leaders of each work group, and modified following a plenary discussion and vote using an electronic voting system. All participants expressed their agreement with each statement, ranking them on a scale of 1 (complete disagreement) to 9 (complete agreement). Statements were accepted only when all the agreement scores were between 7 and 9, otherwise they were reformulated and subjected to a new vote.

\subsection{Management of HIV/HCV in Co-Infected Patients}

Chronic hepatitis $\mathrm{C}$ is characterized by slow progression of fibrosis towards cirrhosis, triggered by liver inflammation; the evolution of cirrhosis towards decompensation due to progressive portal hypertension and failure of liver function; and/or the onset of hepatocellular carcinoma (HCC). Hence, accurate staging of the liver disease, preventative measures, and early diagnosis of such complacations are vital in HIV patients co-infected with $\mathrm{HCV}$.

\subsubsection{Assessment of Liver Disease Stage}

Liver biopsy is still the gold standard for fibrosis staging, on condition that the liver fragment obtained is greater than 20 - $25 \mathrm{~mm}$ length and contains more than 10 portal tracts. However, biopsy is an invasive and expensive procedure that may on occasion be associated with severe complications, and is also prone to measurement error [3]. In contrast, non-invasive strategies have various advantages including lower cost and higher patient acceptability, making them easier to repeat after short intervals. Moreover, several studies have shown that non-invasive methods have similar prognostic accuracy

Table 1. Levels of evidence and scoring of recommendations.

\begin{tabular}{cc}
\hline Strength of recommendation & Quality of evidence \\
\hline A-Good & I-Properly randomized controlled trial \\
B-Moderate & II- Other published studies \\
C-Poor & III-Expert Opinion \\
\hline
\end{tabular}


in $\mathrm{HCV} / \mathrm{HIV}$ co-infection and in patients with chronic hepatitis $\mathrm{C}$ alone. Biochemical parameters, namely the AST-to-platelet ratio index, FIB-4 score, FibroTest, FibroMeter, Hepascore and Zeng's score, correctly stage fibrosis in the majority of patients and generally show high negative predictive values [4]. Likewise, liver stiffness measured by transient elastography (Fibroscan ${ }^{\circledR}$ ) provides the same diagnostic accuracy in HIV/HCV coinfection and $\mathrm{HCV}$ mono-infection. In addition, liver stiffness seems to be a very useful prognostic marker for monitoring the progression of fibrosis over time, which is accelerated in co-infected HIV/HCV subjects [5].

Statement: although liver biopsy remains the gold standard for detecting fibrosis in HIV/HCV co-infected patients, non-invasive assays such as transient elastography and/or biochemical surrogate markers can be used in co-infected HIV/HCV patients, and are particularly useful for monitoring the progression of fibrosis over time (BII).

The guidelines for treatment of complications in cirrhosis patients in the general population can, for the moment, be applied to cirrhotic PLWHIV. Although HCC is an increasing cause of death in these patients and data appears to show it progresses more rapidly in PLWHIV, this finding is controversial, and there is not yet enough data available to support different screening and management procedures for HCC in PLWHIV [6,7].

Statement: early detection and management of complications due to portal hypertension and ESLD in co-infected HCV/HIV patients is vital and should follow the same guidelines as for HCV mono-infected patients. Ultrasound examination at 6-month intervals is the mainstay for hepatocellular carcinoma surveillance (AI).

\subsubsection{End Stage Liver Disease and Liver Transplantation}

It has been demonstrated that permanent discontinuation of cART in compensated cirrhotic patients has a significant link with liver decompensation and shorter survival times. Moreover, increased survival after the first episode of decompensation has been demonstrated in patients taking cART with respect to those discontinuing antiretroviral treatments [8-10]. However, it has not been demonstrated that antiretroviral therapy is able to modify the risk of HCC in patients with HCV-related cirrhosis [11].

Statement: highly active antiretrovirals should be maintained in cirrhotic co-infected HCV/HIV patients to avoid accelerated progression to ESLD, particularly when decompensation occurs, to reduce the incidence of liver-related mortality (AII).

Liver transplantation is currently a treatment option for HIV patients with ESLD or HCC, and in many countries, including Italy, HIV-infected patients have been given access to the national transplant programme since 2002, subject to the strict inclusion and exclusion criteria. However, in HCV/HIV co-infection, transplant outcomes are worse than those reported for HIV-negative patients, mainly due to more severe recurrence of $\mathrm{HCV}$. In fact, recent studies have shown that survival after transplant may be up to $40 \%$ worse in co-infected HIV/HCV patients than in patients with $\mathrm{HCV}$ infection alone. Nevertheless, survival at 5 years post-transplant seems to be around $50 \%$ in HCV-infected PLWHIV with [12-20]. CD4 < 200, high MELD score at transplant, advanced donor age, HCV genotype-1 infection, and double liverkidney transplantation have all been described as predictors of poor prognosis.

Statement: co-infected HIV/HCV patients with ESLD or HCC should be periodically assessed for their eligibility for liver transplantation (B III). Orthotopic liver transplant can be offered to selected HIV/HCV co-infected patients if they meet the eligibility criteria established by national transplant programmes. (B II).

After transplant, the management of immunosuppressive therapy may be particularly troublesome in HIVinfected patients due to its interaction with antiretroviral drugs. Such drug-drug interactions require frequent drug dosage adjustments and monitoring (over- or underdosing may increase the risk of drug-induced toxicity, transplant rejection and/or HIV viral rebound) [21].

Statement: to improve the outcome in co-infected HIV-HCV patients undergoing orthotopic liver transplantation (OLT), both pre transplant and post-transplant cART should be managed by a multidisciplinary team involving pharmacologists and infectious disease specialists with expertise in OLT in HIV-infected patients (AII).

\subsection{Optimized cART in Co-Infected HIV/HCV Patients Not Eligible for PegIFN/RBV Treatment, or in Those Awaiting New Anti-HCV Treatment Options}

Antiretroviral therapy has the well-known advantage of slowing down hepatic disease progression. However, liver disease progression can be accelerated by druginduced liver injury (DILI), which is more common in HCV co-infected PLWHIV. Acute and subacute DILI can be related to immune restoration, and chronic DILI can arise due to cART-induced hepatotoxicity and metabolic changes. DILI has been reported to occur in $15 \%$ $20 \%$ of $\mathrm{HIV} / \mathrm{HCV}$ co-infected individuals. When cART was first introduced, these patients had an up to eightfold risk of severe hepatic damage after starting cART with respect to patients with HIV alone. Interestingly, a higher risk of hepatotoxicity has been reported in patients carrying genotype $3 \mathrm{HCV}$ with respect to the other genotypes $[22,23]$. 


\subsubsection{DILI during Immune Reconstitution Inflammatory Syndrome (IRIS)}

An increase in anti-HCV specific immune response after cART initiation may lead to higher levels of transaminase following a sudden increase in CD4+ T-cell count from a low baseline value [24-27]. DILI during IRIS is more frequent in advanced naïve patients who started cART with drugs able to dramatically decrease the HIV viral load.

Statement: to reduce the risk of IRIS, cART should be started earlier in co-infected HCV/HIV persons (AIII). If patients have a high risk of developing DILI due to IRIS (CD4+ T-cell count level $<200$ cell $/ \mathrm{mm} 3$, moderatesevere liver disease and/or HCV genotype 3), they should be closely monitored, especially when fast-acting viral suppressors are prescribed (BIII).

\subsubsection{DILI Due to Direct Hepatotoxicty}

First and foremost, it is important to note that this type of toxicity differs from immune allergic reactions involving the liver, which are not related to HCV status. Indeed, NRTI hepatotoxicityis linked to depletion in mitochondrial DNA, mainly caused by the thymidine analogue reverse-transcriptase inhibitors (NRTI) [28,29]. Being less toxic to the mitochondria, abacavir, tenofovir, lamivudine and emtricitabine are safer options in chronic liver disease [30]. NNRTIs also present a high risk of DILI. Extensive data are recorded for efavirenz and nevirapine, [31] and the latter seems to cause a higher risk of DILI than the former [31,32], in particular when given once daily [33]. However, direct NNRTI-related DILI usually occurs beyond the third month of treatment, and an increased cumulative incidence has been reported for nevirapine [32]. It is thought that etravirine and rilpivirine may grant better liver safety as compared to the older drugs of the same class, but little data is available at the present time [33].

Tipranavir and higher doses of ritonavir have been associated with an increased incidence of DILI [34,35], but to date, hepatic safety of darunavir in HIV/HCV co-infected patients has not been extensively investigated. Nevertheless, a study conducted on HIV-infected patients, with or without HCV co-infection, enrolled in the Italian darunavir EAP showed a moderate increase in transaminases in co-infected subjects, whether cirrhosis was present or not. However, it is not clear whether or not this increase was linked to the presence of the $200 \mathrm{mg}$ ritonavir boosting [36]. It is known, however, that the pharmacokinetics of darunavir/ritonavir $600 \mathrm{mg} / 100 \mathrm{mg}$ twice daily are not affected by mild (Child-Pugh classification 1) or moderate (Child-Pugh classification 2) hepatic impairment [37,38]. Furthermore, unboosted atazanavir $400 \mathrm{mg}$ QD and fosamprenavir $1400 \mathrm{mg}$ QD, boosted or unboosted by ritonavir, are commonly used with satisfying results in co-infected $\mathrm{HIV} / \mathrm{HCV}$ subjects when TDM is available [39,40]. Moreover, registrative trials have demonstrated that raltegravir may produce an increase in AST/ALT of 3 - 4 degrees, but this occurred in no more than $2 \%-3 \%$ of the treated population. Data on co-infected populations are still scarce, although a study conducted on 135 subjects treated with integrase inhibitor, 45 of whom were co-infected by HCV, showed that the risk of a 1 - 2 degree increase in transaminases was higher in co-infected $(39.5 \%)$ vs. mono-infected HIV patients $(9.1 \%)$. This was especially true when cholesterol was $\geq 200 \mathrm{mg} / \mathrm{dl}$ and raltegravir was co-administered with a protease inhibitor. In subjects with advanced hepatic disease, the AST/ALT increase was not signifycantly higher $[41,42]$.

Although hepatic toxicity events have been reported for some CCR5 inhibitors, the MOTIVATE trial revealed the considerable hepatic safety of maraviroc - after 48 weeks the AST/ALT rate increase to $3-4$ degree was $0.6 \%-3.7 \%[43,44]$. Studies to evaluate the anti-inflammatory and anti-fibrogenic effect of CCR5 inhibition with maraviroc are ongoing [45].

Statement: didanosine and stavudine should be avoided in co-infected HCV/HIV patients due to a risk of lactic acidosis (AII). NNRTI prescription should be individualized, and discouraged in those with grade $3-4$ transaminase elevation at baseline (BII). In general, nevirapine prescription should be discouraged in naïve patients (BIII), and is contraindicated at CD4 cell counts $>400$ in males and $>250 / \mathrm{ml}$ in females. Limited data appear to indicate etravirine and rilpivirine for co-infected HIV/HCV patients (C I). PIs should be boosted with 100 $\mathrm{mg} /$ day of ritonavir (but no more than $200 \mathrm{mg} /$ day) (AI-II), although unboosted PIs are a reasonable means of optimizing cART in co-infected HIV/HCV patients. (AII) TDM, when available, should be used to monitor the correct dosage of NNRTIs and boosted or unboosted PIs. (AII) Raltegravir and maraviroc should be considered as safe options for co-infected HIV/HCV patients with mild to moderate hepatic disease (AII).

\subsubsection{DILI Due to Metabolic Toxicity}

Changes in glucose and lipid metabolism contribute to the progression of hepatic fibrosis in patients with chronic hepatitis $\mathrm{C}$ through liver steatosis. This has also been reported in as much as $61 \%$ of $\mathrm{HIV} / \mathrm{HCV}$ patients, in whom it is associated with a higher degree of liver fibrosis [46]. Steatosis pathogenesis is multifactorial in these patients, but the metabolic effects of some antiretroviral drugs may contribute to liver steatosis and NASH, as both cause and effect of insulin resistance and increase in leptin plasma levels [47]. Indeed, long-term exposure to NRTIs and especially to "d-Drugs" is associated with insulin resistance, diabetes and increased serum lactates 
[48]. Early-generation protease inhibitors also induced insulin resistance, as well as increasing triglycerides, total cholesterol, leptin and peptide $\mathrm{C}$, while reducing HDL cholesterol and adiponectin [47]. Even though studies on the effect of many antiretrovirals in current use on liver steatosis are lacking, lipodystrophy and a high HOMA score have both displayed a significant link with advanced liver fibrosis [49]. Raltegravir showed a neutral metabolic effect in clinical studies [50].

Statement: mitochondrial toxicity and insulin resistance appear to play a key role in cART-related liver steatosis, NASH, and consequently liver fibrosis progression. Highly mitochondria-toxic antiretroviral drugs (AZT, DDI, D4T) should therefore be avoided (AII). Drugs with major effects on glucose metabolism also need to be used with caution (BII).

\subsection{4. cART in Co-Infected HIV/HCV Patients with Co-Morbidities}

A meta-analysis of 24 observational retrospective studies and several clinical trials found that HIV/HCV co-infection is associated with increased risk of kidney disease, including proteinuria and renal failure [51]. The largest study of biopsy-proven renal disease has found that $\mathrm{HIV} / \mathrm{HCV}$-associated nephropathies reduced survival as compared to HCV mono-infection [52]. Furthermore, the 10 -year incidence of bone fractures was 3.6-fold higher in $\mathrm{HCV}$ co-infected patients, probably because patients with chronic liver disease have decreased BMD [53].

It has also been demonstrated that co-infected HIV/ HCV patients more frequently suffer from neurologic disturbances than mono-infected HIV patients [54] and also have higher rates of global cognitive impairment, especially in learning and memory [55]. Sensory neuropathy is a common complication of HIV infection, may be worsened by cART-related toxicity, and is associated with HCV infection, raising concerns about possible synergistic effects of these viruses on the peripheral nervous system [56].

Statement: the renal function, bone metabolism, cardiovascular system and central and peripheral nervous systems of co-infected HIV/HCV patients may be affected by extra-hepatic manifestations of HCV. Therefore, in addition to appropriate screening and close follow-up of co-morbidities, the following recommendations apply:

1) An early switch to drugs with lower renal and bone toxicity when signs of impaired renal function and/or bone metabolism are detected (A-BI).

2) In co-infected HIV/HCV persons with cardiovascular risk, no different cART options are recommended with respect to mono-infected HIV patients (AIII).

3) Patients with minor neurocognitive impairment (HAND) on efavirenz should receive close individual- ized follow-up (BIII).

\subsection{5. cART in Patients with ESLD}

Patients with ESLD need to be treated by a multidisciplinary team (AIII). Antiretroviral treatment interruptions are an additional risk factor for accelerated cirrhosis progression and liver-related mortality in $\mathrm{HIV} / \mathrm{HCV}$ coinfection [57,58]. PLWHIV with concomitant ESLD in particular need continued effective antiretroviral therapy. However, this issue is complicated by the fact that some antiretroviral drugs require dosage adjustments to compensate for reduced hepatic drug metabolism in ESLD [59].

Statement: co-infected HIV/HCV patients with ESLD should be maintained on cART (AII). No drugs are formally indicated in patients with a Child-Pugh score of C - limited data are available only for fosamprenavir 300 BID plus ritonavir $100 \mathrm{mg} / \mathrm{QD}$. TDM is highly recommended to optimize the dosage in patients with ESLD (AII). Off-label use and dosage of antiretroviral drugs should be considered only when supported by TDM. However, since TDM data are not validated in ESLD patients, any therapeutic decision should be based on immuno-virological and safety data (AII).

\subsection{Optimized c-ART in Co-Infected HIV/HCV Patients during Treatment with Peginterferon plus Ribavirin}

HCV- and HIV-infection highly influence each other's progression. New data aimed at clarifying the pathophysiological mechanisms underlying this interaction strongly support the importance of early treatment of both infections [60-63].

\subsubsection{HCV or HIV Therapy: What to Start First?}

Both Italian and European guidelines recommend that antiretroviral treatment should precede anti-HCV treatment in patients with a supposed rapid progression of HIV disease (HIV-RNA $>100,000$ copies/mL or a CD4+ T-cells decay $>100$ cells/uL in the previous year) at any level of CD4 cell count, and in the case of CD4+ T-cell counts lower than 500 cells/uL because it may help attain a sustained virological response (SVR) [64,65]. Indeed, data from a large observational Italian study on treatment of HCV infection in HIV-infected patients seem to support an earlier start of cART in co-infected subjects undergoing standard anti-HCV treatment (based on peg Interferon and ribavirin), as suppression of HIV-RNA was significantly associated with "eradication" (SVR) of HCV infection [66].

Statement: the eradication of HCV (sustained virological response-SVR) is of huge importance in co-infected HIV/HCV patients. Nevertheless, in subjects with detectable HIV-RNA and a stable CD4+ T-cell count, even 
higher than $500 / \mathrm{uL}$, the start of cART is recommended before commencing standard anti-HCV treatment. (BII) In co-infected $\mathrm{HIV} / \mathrm{HCV}$ patients eligible for standard anti-HCV treatment with a CD4 + T-cell count between 350 and 500/uL, cART should be commenced before anti$\mathrm{HCV}$ treatment (BII). In patients not eligible for antiHCV treatment, cART must be started immediately due to its protective effect on the progression of liver fibrosis (AII).

A stable immune recovery is desirable before commencing anti-HCV treatment (AII).

Co-infected HIV/HCV patients with undetectable HIV-RNA, CD4 + T-cell count $<200 / \mathrm{uL}$ and advanced liver disease should be treated with anti-HCV drugs, despite the lack of data in profoundly immunosuppressed patients (AII).

\subsubsection{The Influence of cART on the Outcome of Standard Anti-HCV Treatment}

The influence of cART on the outcome of standard anti$\mathrm{HCV}$ treatment has not yet been fully clarified, and the data that is available comes mainly from retrospective analyses. Most of these studies suggest that cART has no impact on the achievement of SVR, with the exception of drugs that amplify the adverse effects of PegIFN/RBV (like zidovudine, stavudine or didanosine) [67,68]. Several studies have raised concerns about the potential negative interaction between abacavir and ribavirin, showing a significantly reduced rate of SVR in patients taking abacavir, mainly when ribavirin has to be administered at reduced doses [69]. However, the postulated competition between abacavir and ribavirin for intracellular phosphorylation was not confirmed by an in vitro study [69]. Moreover, this impact of abacavir was not confirmed in other cohorts of co-infected HIV/HCV patients treated with PegIFN and weight-based ribavirin [70].

Some antiretroviral drugs induce insulin resistance, which has emerged as an important predictor of rapid virological response (RVR) and SVR in many studies, and this should be taken into account when selecting cART, even though to date there have been no trials to determine whether an insulin-resistance-guided shift of antiretroviral drugs may have a favourable effect on SVR [71,72].

Finally, drug-drug interactions must be carefully considered, especially in direct-acting antivirals (DAAs such as HCV-protease and polymerase inhibitors) [73-75]. To this end, several studies have described drug - drug interactions between boceprevir, telaprevir and antiretrovirals in normal subjects. The results of these studies are summarized in Table 2, which also reports which antiretrovirals can be given to candidates for treatment with boceprevir and telaprevir. That being said, pilot studies in which boceprevir was administered to patients taking anti-HIV protease inhibitors failed to confirm the potential clinical significance of these interactions. No information is yet available regarding the new anti-HCV agents currently in phase II and III trials in HIV-infected patients, but drug - drug interactions between all classes of anti HCV DAA and antiretrovirals may be significant, with the possible exception of nucleosides/nucleotide polymerase inhibitors.

Statement: in co-infected HIV/HCV candidates for both antiretroviral and anti-HCV treatment, it is advisable to start cART, or to modify it if required, at least 4 - 8 weeks before starting anti-HCV therapy to take into account the potential interactions between antiretroviral drugs, PegIFN/RBV and the new DAAs. This will enable better management of any side effects caused by the cART regimen, giving the patient time to adapt (AIII).

Statement: In HIV/HCV co-infected candidates for anti-HCV treatment, the cART regimen should have the following characteristics:

- Efficacy: general principles for selecting cART in HIV-mono-infected subjects should be followed (AIII).

- Pharmacodynamic interactions potentially causing an amplification of side effects of pegylated interferon and/or ribavirin need to be ruled out.

- $\quad \mathrm{ddI} \pm \mathrm{d} 4 \mathrm{~T}$ must be avoided because the combined use of these drugs with RBV increases the risk of mitochondrial toxicity (pancreatitis, lactic acidosis) (AI).

- AZT should be avoided due to an increased risk of myelotoxicity (anaemia, neutropenia) (BI).

- EFV and boosted ATV may be used, but close monitoring is advised as EFV may amplify the neuropsychiatric effects of pegylated interferon, while ATV, when given in combination with RBV, may increase levels of plasma bilirubin (CII).

- Drugs with the lowest impact on insulin sensitivity should be chosen (BIII).

- New ARV drugs with a low metabolic impact can be prescribed, but should currently be used with caution as so far there is no published clinical data concerning their use in anti HCV-treatment (clinical trials to study their use in this particular setting are recommended) (CIII).

- In candidates for boceprevir and/or telaprevir, cART regimen should be switched to one without significant drug - drug interactions at least $4-8$ weeks before starting anti-HCV therapy whenever possible (Table 2). In patients whose cART regimen cannot be altered, three mutually exclusive scenarios should be considered on an individual basis:

Delay of anti-HCV therapy in patients with mild fibrosis Cautious use of darunavir in telaprevir candidates and cautious use of atazanavir in boceprevir candidates, 
Table 2. Summary of recommendations for antiretroviral drug administration in candidates for anti-HCV treatment.

\begin{tabular}{|c|c|c|c|c|}
\hline $\begin{array}{l}\text { Antiretroviral } \\
\text { drug class }\end{array}$ & Drug & $\begin{array}{l}\text { Combination with } \\
\text { PegIFN + RBV }\end{array}$ & $\begin{array}{c}\text { Combination with PegIFN + RBV } \\
+ \text { telaprevir }\end{array}$ & $\begin{array}{c}\text { Combination with PegIFN + } \\
\text { RBV + BOC }\end{array}$ \\
\hline \multirow{4}{*}{ NRTI } & Zidovudine, Stavudine or Didanosine & \multicolumn{3}{|c|}{ No additional toxicity } \\
\hline & Abacavir & \multicolumn{3}{|c|}{ Consider alternative treatment for use with low ribavirin doses } \\
\hline & Lamivudine or Emtricitabine & \multicolumn{3}{|c|}{ Can be used } \\
\hline & Tenofovir & \multicolumn{3}{|c|}{ Can be used but eGFR should be monitored (increased exposure if not used with efavirenz) } \\
\hline \multirow{4}{*}{ PI } & Lopinavir + Ritonavir & Can be used & \multicolumn{2}{|c|}{ Not advised: significant DDI } \\
\hline & Darunavir + Ritonavir & Can be used & $\begin{array}{l}\text { Not advised but could be considered in } \\
\text { selected patients with no alternatives }\end{array}$ & Not advised \\
\hline & Atazanavir + Ritonavir & Can be used & Can be used & $\begin{array}{l}\text { Not advised but could be } \\
\text { considered in selected patients } \\
\text { with no alternatives }\end{array}$ \\
\hline & Tipranavir + Ritonavir & Can be used & Not advised due to potential inter & actions_- no data on DDI \\
\hline \multirow{4}{*}{ NNRTI } & Efavirenz & Can be used & $\begin{array}{l}\text { Can be used with TPV } 1125 \text { t.i.d. } \\
\text { (consider additional costs) }\end{array}$ & Not advised \\
\hline & Nevirapine & Can be used & \multicolumn{2}{|c|}{ Not advised (potential interactions - data on DDI) } \\
\hline & Rilpivirine & Can be used & $\begin{array}{c}\text { Can be used but QT interval } \\
\text { monitoring is advised }\end{array}$ & Not advised (no data on DDI) \\
\hline & Etravirine & & Can be used & \\
\hline \multirow{2}{*}{ Others } & Raltegravir & \multicolumn{3}{|c|}{ Can be used } \\
\hline & Maraviroc & Can be used & \multicolumn{2}{|c|}{ Not advised (no data on DDI) } \\
\hline
\end{tabular}

without other "allowed" anti-HIV treatment options.

Withdrawal of cART for three months in candidates for telaprevir with CD4 > 500 and a CD4 nadir greater than 350 .

\section{Conclusion}

While we await new anti-HCV drugs, which may consent interferon-free, all-oral, pangenotypic, and 100\% effective treatment of $\mathrm{HCV}$, optimization of antiretroviral regimens may play a pivotal role in stabilizing and even improving the course of HCV co-infection in PLWHIV candidates for anti-HCV treatment and patients with advanced liver disease.

\section{REFERENCES}

[1] J. A. Pineda, M. R. Gomez, F. D. Garcia, et al., "HIV Coinfection Shortens the Survival of Patients with Hepatitis C Virus Related Decompensated Cirrhosis," Нераtology, Vol. 41, No. 4, 2005, pp. 779-789. doi:10.1002/hep.20626

[2] M. Puoti, R. Rossotti, A. Garlaschelli and R. Bruno, "Hepatocellular Carcinoma in HIV Hepatitis C Virus," Current Opinion in HIV and AIDS, Vol. 6, No. 1, 2011, pp. 534-538. doi:10.1097/COH.0b013e32834bd2b7

[3] E. Strauss, "Usefulness of Liver Biopsy in Chronic Hepatitis C," Annals of Hepatology, Vol. 9, 2010, pp. 39-42.

[4] P. Calès, P. Halfon, D. Batisse, et al., "Comparison of
Liver Fibrosis Blood Tests Developed for HCV with New Specific Tests in HIV/HCV Co-Infection," Journal of Hepatology, Vol. 53, No. 2, 2010, pp. 238-244.

[5] M. Sánchez-Conde, M. L. Montes-Ramírez, P. Miralles, et al., "Comparison of Transient Elastography and Liver Biopsy for the Assessment of Liver Fibrosis in HIV/ Hepatitis C Virus-Coinfected Patients and Correlation with Noninvasive Serum Markers," Journal of Viral Hepatitis, Vol. 17, No. 4, 2010, pp. 280-286. doi:10.1111/j.1365-2893.2009.01180.x

[6] E. Garlassi, P. Ventura, P. Beretta, et al., "Hepato Cellular Carcinoma in HIV-Infected Patients: Check Early, Treat Hard," Infection, Vol. 38, Suppl. 1, 2010, pp. 82182.

[7] K. Prestini, L. Biasi, M. Mendeni, et al., "Incidence and Predictors of Hepatocellular Carcinoma (HCC) in Cirrhotics Living with HIV (Abstract)," 5th International HIV and Hepatitis Workshop, Lisbon, 4-6 June 2009.

[8] N. Qurishi, C. Kreuzberg, G. Luchters, et al., "Effect of Antiretroviral Therapy on Liver Related Mortality in Patients with HIV and Hepatitis C Virus Coinfection," Lancet, Vol. 362, No. 9397, 2003, pp. 1708-1713. doi:10.1016/S0140-6736(03)14844-1

[9] J. A. Pineda, J. A. García-García, M. Aguilar-Guisado, et al., "Clinical Progression of Hepatitis C Virus-Related Chronic Liver Disease in Human Immunodeficiency VirusInfected Patients Undergoing Highly Active Antiretroviral Therapy," Hepatology, Vol. 46, No. 3, 2007, pp. 622630.

[10] M. Lopez-Dieguez, M. L. Montes, J. F. Pascual Pereja, et 
al., "The Natural History of Liver Cirrhosis in HIVHepatitis C Virus-Coinfected Patients," AIDS, Vol. 25, No. 7, 2011, pp. 899-904. doi:10.1097/QAD.0b013e3283454174

[11] M. Saves, S. Vandentorren, V. Daucourt, et al., "Severe Hepatic Cytolysis: Incidence and Risk Factors in Patients Treated by Antiretroviral Combinations. Aquitaine Cohort, France, 1996-1998," AIDS, Vol. 13, No. 17, 1999 , pp. F115-F121. doi:10.1097/00002030-199912030-00002

[12] M. V. Ragni, B. Eghttesad, K. W. Schlesinger, et al., "Pretransplant Survival Is Shorter in HIV-Positive than HIV-Negative Subjects with End-Stage Liver Disease," Liver Transplant, Vol. 11, No. 11, 2005, pp. 1425-1430. doi:10.1002/lt.20534

[13] Y. Sugawara, S. Tamura and N. Kokudo, "Liver Transplantation in HCV/HIV Positive Patients," World Journal of Gastrointestinal Surgery, Vol. 3, No. 2, 2011, pp. 2128. doi:10.4240/wjgs.v3.i2.21

[14] M. V. Ragni, S. H. Belle, K. Im, et al., "Survival of Human Immunodeficiency Virus Infected Liver Transplant Recipients," The Journal of Infectious Diseases, Vol. 188, No. 10, 2003, pp. 1412-1420. doi:10.1086/379254

[15] G. W. Neff, A. Bonham, A. G. Tzakis, et al., "Orthtopic Liver Transplantation in Patients with Human Immunodeficiency Virus and End-Stage Liver Disease," Liver Transplant, Vol. 9, No. 3, 2003, pp. 239-247. doi:10.1053/jlts.2003.50054

[16] M. E. de Vera, I. Dvorchik, K. Tom, et al., "Survival of Liver Transplant Patients Coinfected with HIV and HCV Is Adversely Impacted by Recurrent Hepatitis C," American Journal of Transplantation, Vol. 6, No. 12, 2006, pp. 2983-2993. doi:10.1111/j.1600-6143.2006.01546.x

[17] I. Schreibman, J. J. Gaynor, D. Jayaweera, et al., "Outcomes after Orthotopic Liver Transplantation in 15 HIVInfected Patients," Transplantation, Vol. 84, No. 6, 2007, pp. 697-705. doi:10.1097/01.tp.0000282873.24648.5b

[18] J. Miro, M. Montejo, L. Castells, et al., "Prognostic Factors of Mortality in HCV/HIV Coinfected Liver Transplant Recipients from the FIPSE OLT-HIV-05- GESIDA 45-05 Cohort Study (2002-2006)," 15th Conference on Retroviruses and Opportunistic, Boston, 3-6 February 2008, p. 177.

[19] A. L. Mindikoglu, A. Regev and L. S. Magder, "Impact of Human Immunodeficiency Virus on Survival after Liver Transplantation: Analysis of United Network for Organ Sharing Database," Transplantation, Vol. 85, No. 3, 2008, pp. 359-368. doi:10.1097/TP.0b013e3181605fda

[20] J. C. Duclos-Vallee, C. Feray, M. Sebagh, et al., "Survival and Recurrence of Hepatitis $\mathrm{C}$ after Liver Transplantation in Patients Coinfected with Human Immunodeficiency Virus and Hepatitis C Virus," Hepatology, Vol. 47, No. 2, 2008, pp. 407-417. doi:10.1002/hep. 21990

[21] K. Marfo and S. Greenstein, "Antiretroviral and Immunosuppressive Drug-Drug Interactions in Human Immunodeficiency Virus-Infected Liver and Kidney Transplant Recipients," Transplantation Proceedings, Vol. 41, No. 9, 2009, pp. 3796-3799. doi:10.1016/i.transproceed.2009.06.186

[22] V. Soriano, M. Puoti, P. Garcia-Gascó, J. K. Rockstroh,
Y. Benhamou, P. Barreiro and B. McGovern, "Antiretroviral Drugs and Liver Injury," AIDS, Vol., 22, No. 1, 2008, pp. 1-13. doi:10.1097/QAD.0b013e3282f0e2fd

[23] I. Maida, S. Babudieri, C. Selva, et al., "Liver Enzyme Elevation in Hepatitis C Virus (HCV)-HIV-Coinfected Patients Prior and after Initiation of CART: Role of HCV Genotypes," AAIDS Research and Human Retroviruses, Vol. 22, No. 2, 2006, pp. 139-143. doi:10.1089/aid.2006.22.139

[24] M. John, J. Flexman and M. A. H. French, "Hepatitis C Virus-Associated Hepatitis Following Treatment of HIVInfected Patients with HIV Protease Inhibitors: An 'Immune Restoration Disease'?” AIDS, Vol. 12, No. 17, 1998, pp. 2289-2293. doi:10.1097/00002030-199817000-00010

[25] M. A. French, N. Lenzo, M. John, et al., "Immune Restoration Disease after the Treatment of Immunodeficient HIV-Infected Patients with Highly Active Antiretroviral Therapy," HIV Medicine, Vol. 1, No. 2, 2001, pp. $107-$ 115.

[26] I. Ofotokun, S. E. Smithson, C. Lu, et al., "Liver Enzyme Elevation and Immune Reconstitution among Treatment Naive HIV-Infected Patients Instituting Antiretroviral Therapy," The American Journal of the Medical Sciences, Vol. 334, No. 5, 2007, pp. 334-341. doi:10.1097/MAJ.0b013e31811ec780

[27] S. F. Stone, S. Lee, N. M. Keane, et al., "Association of Increased Hepatitis C Virus (HCV)-Specific IgG and Soluble CD26 Dipeptidyl Peptidase IV Enzyme Activity with Hepatotoxicity after Highly Active Antiretroviral Therapy in Human Immunodeficiency Virus-HCV-Coinfected Patients," The Journal of Infectious Diseases, Vol. 186, No. No. 10, 2002, pp. 1498-1502.

[28] G. Moyle, "Mechanism of HIV and Nucleoside Reverse Transcriptase Inhibitor Injury to Mitochondria," Antiviral Therapy, Vol. 10, Suppl. 2, 2005, pp. M47- M52.

[29] A. Igoudjil, A. Abbey-Toby, K. Begriche, et al., "High Doses of Stavudine Induce Fat Wasting and Mild Liver Damage without Impairing Mitochon Drial Respiration in Mice," Antiviral Therapy, Vol. 12, No. 3, 2007, pp. 389400.

[30] P. Braitstein, A. Palepu, D. Dietrich, et al., "Special Considerations in the Initiation and Management of Antiretroviral Therapy in Individuals Coinfected with HIV and Hepatitis C," AIDS, Vol. 18, No. 17, 2004, pp. 2221-2234. doi:10.1097/00002030-200411190-00002

[31] Z. S. Lamar and M. Nunez, "Higher Risk of Severe DrugInduced Liver Injury among Hispanic HIV-Infected Patients after Initiation of Highly Active Antiretroviral Therapy," journal of the International Association of Providers of AIDS Care (JIAPAC), Vol. 10, No. 3, 2011, pp. 183-186. doi:10.1177/1545109711401411

[32] L. Martın-CarboneroL, M. Nunez, J. Gonzalez-Lahoz and V. Soriano, "Incidence of Liver Injury after Beginning Antiretroviral Therapy with Efavirenz or Nevirapine," HIV/AIDS Clinical Trials, Vol. 4, No. 2, 2003, pp. 115120. doi:10.1310/N4VT-3E9U-4BKN-CRPW

[33] F. Vanleth, P. Phanuphak, K. Ruxrungtham, et al., "Comparison of first-Line Antiretroviral Therapy with Regimens including Nevirapine, Efavirenz, or Both Drugs 
plus Stavudine and Lamivudine: A Randomized OpenLabel Trial, the 2NN Study," Lancet, Vol. 363, No. 9417 , 2004, pp. 1253-1263. doi:10.1016/S0140-6736(04)15997-7

[34] B. Clotet, N. Clumeck, C. Katlama, et al., "Safety of Etravirine in HIV-1/Hepatitis B and/or C Virus Co-Infected patients: Pooled 96 Week Results from the Phase III DUET Trials," Journal of Antimicrobial Chemotherapy, Vol. 65, No. 11, 2010, pp. 2450-2454. doi: $10.1093 / \mathrm{jac} / \mathrm{dkq} 332$

[35] M. Sulkowski, S. Mehta, R. Chaisson, et al., "Hepatotoxicity Associated with Protease Inhibitor-Based Antiretroviral Regimens with or without Concurrent Ritonavir," AIDS, Vol. 18, No. 17, 2004, pp. 2277-2284. doi:10.1097/00002030-200411190-00008

[36] J. C. Gathe Jr, G. Pierone, P. Piliero, et al., "Efficacy and Safety of Three Doses of Tipranavir Boosted with Ritonavir in Treatment-Experienced HIV Type-1 Infected Patients," AIDS Research Human Retroviruses, Vol. 23, No. 2, 2007, pp. 216-223. doi:10.1089/aid.2006.0178

[37] K. McKeage, C. M. Perry, S. J. Keam, et al., "Darunavir: A Review of Its Use in the Management of HIV Infection in Adults," Drugs, Vol. 69, No. 4, 2009, pp. 477-503. doi:10.2165/00003495-200969040-00007

[38] V. Sekar, S Spinosa-Guzman, E. De Paepe, et al., "Pharmacokinetics of Multiple-Dose Darunavir in Combination with Low-Dose Ritonavir in Individuals with Mild-toModerate Hepatic Impairment," Clinical Pharmacokinetics, Vol. 49, No. 5, 2010, pp. 343-350. doi:10.2165/11530690-000000000-00000

[39] A. Rachilis, Clotet B, J. Baxter, et al., "Safety, Tolerability, and Efficacy of Darunavir (TMC114) with LowDose Ritonavir in Treatment-Experienced, Hepatitis B or C Co-Infected Patients in POWER 1 and 3," HIV Clinical Trials, Vol. 8, No. 4, 2007, pp. 213-220. doi:10.1310/hct0804-213

[40] M. Regazzi, P. Villani, R. Gulminetti, et al., "Therapeutic Monitoring and Variability of Atazanavir in HIV-Infected Patients, with and without HCV Coinfection, Receiving Boosted or Unboosted Regimens," Therapeutic Drug Monitoring, Vol. 33, No. 3, 2011, pp. 303-308.

[41] F. Gatti, P. Nasta, A. Loregian, et al., "Unboosted Fosamprenavir Is Associated with Low Drug Exposure in HIV-1 Infected Patients with Mild Moderate Liver Impairment Resulting from HCV Related Cirrhosis," Journal of Antimicrobial Chemotherapy, Vol. 63, No. 3, 2009, pp. 575578. doi:10.1093/jac/dkn525

[42] P. Nasta, F. Gatti, F. Borghi, et al., "Raltegravir He patic Tolerability in Patients with or without HIV/HCV CoInfection," Fifth Workshop on HIV and Hepatitis Co-Infection, 19-21 June, 2009, Lisbon, Abs. 38.

[43] J. Macias, K. Neukam, J. Poetilla and J. A. Jribarren (de Los Santos and al for HEPRAL Study Team), "Liver Tolerance of Raltegravir-Containing Antiretroviral Therapy in HIV-Infected Patients with Chronic Hepatitis C," Journal of Antimicrobial Chemotherapy, Vol. 66, No. 6, 2011, pp. 1346-1350.doi:10.1093/jac/dkr083

[44] M. Genebat, E. Ruiz-Mateos, I. Pulido, A. GonzalezSerma, A. Garcia-Perganeda, et al., "Long-term Immuno- virogical Effect and Tolerability of a Maraviroc-Containing Regimen in Routine Clinical Practice," Currrent HIV Research, Vol. 8, No. 6, 2010, pp. 482-486.

[45] S. Abel, J. D. Davis, C. E. Ridgway, et al., "Pharmacokinetics, Safety and Tolerability of a Single Oral Dose of Maraviroc in HIV-Negative Subjects with Mild and Moderate Hepatic Impairment," Antiviral Therapy, Vol. 14, 2009, pp. 831-837. doi:10.3851/IMP1297

[46] P. Nasta, "Immune Activation, Aging and Gender and Progression of Liver Disease," Acta Bio-Medica, Vol. 82 , No. 2, 2011, pp. 115-123.

[47] F. Bani-Sadr, F. Carrat, P. Bedossa, et al., "Hepatic Steatosis in HIV-HCV Coinfected Patients: Analysis of Risk Factors," AIDS, Vol. 20, No. 4, 2006, pp. 525-531. doi:10.1097/01.aids.0000210606.63138.f5

[48] B. H. McGovern, "Hepatic Steatosis in HV/HCV-Coinfected Patients: Time to Reevaluate! Gastroenteroogy, Vol. 140, No. 3, 2011, pp. 809-817. doi:10.1053/j.gastro.2011.01.025

[49] P. Halfon, G. Penaranda, F. Carrat, et al., "Influence of Insulin Resistance on Hepatic Fibrosis and Steatosis in HCV Monoinfected Compared with HIV-HCV Co-Infected Patients," Alimentary Pharmacology \& Therapeutics, Vol. 30, No. 1, 2009, pp. 61-70. doi:10.1111/j.1365-2036.2009.03995.x

[50] M. A. Loko, et al., "Impact of CART Exposure and Associated Lipodystrophy on Advanced Liver Fibrosis in HIV/HCV-Coinfected Patients," Journal of Viral Hepatitis, Vol. 18, 2010, pp. 307-314.

[51] P. Pérez-Matute, L. Pérez-Martínez, J. R. Blanco and J. A. Oteo, "Neutral Actions of Raltegravir on Adipogenesis, Glucose Metabolism and Lipolysis in 3T3-L1 Adipocytes," Current HIV Research, Vol. 9, No. 3, 2011, pp. 74-79. doi: $10.2174 / 157016211795945278$

[52] M. J. Fisher, C. M. Wyat, K. Gordon, et al., "Hepatitis C and the Risk of Kidney Disease and Mortality in Veterand with HIV," Journal of Acquired Immune Deficiency Syndromes, Vol. 53, No. 2, 2010, pp. 222-226. doi:10.1097/QAI.0b013e3181b980d4

[53] H. Izzedine, D. Sene, P. Cacoub, et al., "Kidney Diseases in HIV/HCV Coinfected Patients," AIDS, Vol. 23, No. 10, 2009, pp. 1219-1226. doi:10.1097/QAD.0b013e32832ac36a

[54] F. Collin, X. Duval, V. Le Moing, et al., "Ten Year Incidence and Risk Factor of Bone Fractures in a Cohort of Treated HIV-1 Infected Adults," AIDS, Vol. 23, No. 8, 2009, pp. 1021-1024. doi:10.1097/QAD.0b013e3283292195

[55] C. H. Hinkin, S. A. Castellon, A. J. Levin, et al., "Neurocognition in Individuals Coinfected with HIV and Hepatitis C," Journal of Addictive Diseases, Vol. 27, No. 2, 2008, pp. 11-17. doi:10.1300/J069v27n02_02

[56] H. A. Aronow, A. J. Weston, B. B. Pezeshki and T. S. Lazzarus, "Effects of Coinfection with HIV and Hepatitis C Virus on the Nervous System," The AIDS Reader, Vol. 18, No. 1, 2008, pp. 43-48.

[57] P. Ancuta, A. Karnat, K. J. Kunstman, et al., "Microbial Translocation Is Associated with Increased Monocyte 
Activation and Dementia in AIDS Patient," PLoS One, Vol. 3, No. 6, 2008, e2516. doi:10.1371/journal.pone.0002516

[58] J. Thorpe, S. Saeed, E. E. M. Moodie and M. B. Klein, "For the Canadian Co-Infection Cohort Study (CTN222). Antiretroviral Treatment Interruption Leads to Progression of Liver Fibrosis in HIV-hepatitis C Virus Co-Infection," AIDS, Vol. 25, No. 7, 2011, pp. 967-975. doi:10.1097/QAD.0b013e3283455e4b

[59] M. López-Diéguez, M. L. Montes, J. F. Pascual-Pareja, et al, "The Natural History of Liver Cirrhosis in HIVHepatitis C Virus-Coinfected Patients," AIDS, Vol. 25, No. 7, 2011, pp. 899-904. doi:10.1097/QAD.0b013e3283454174

[60] S. Dominguez, J. Ghoen, G. Peytavin, et al., "Impact of Hepatitis C and Liver Fibrosis on Antiretroviral Plasma Drug Concentrations in HIV-HCV Coinfected Patients The HEPADOSE Study," Journal of Antimicrobial Chemotherapy, Vol. 65, No. 11, 2010, pp 2445-2449. doi: $10.1093 / \mathrm{jac} / \mathrm{dkq} 320$

[61] R. Bruno, S. Galastri, P. Sacchi, et al., "Gp120 Modulates the Biology of Human Hepatic Stellate Cells: A Link between HIV Infection and Liver Fibrogenesis," Gut, Vol. 59, 2010, pp. 513-520. doi:10.1136/gut.2008.163287

[62] A. Kovacs, R. Karim, W. J. Mack, et al., "Activation of CD8 T cells Predicts Progression of HIV Infection in Women Coinfected with Hepatitis C Virus," The Journal of Infectious Diseases, Vol. 201, No. 6, 2010, pp.823-834. doi:10.1086/650997

[63] V. D. Gonzalez, K. Falconer, K. G. Blom, et al., "High Levels of Chronic Immune Activation in the T-Cell Compartments of Patients Coinfected with Hepatitis C Virus and Human Immunodeficiency Virus Type 1 and on Highly Active Antiretroviral Therapy Are Reverted by Alpha Interferon and Ribavirin Treatment," Journal of Virology, Vol. 83, No. 21, 2009, pp.11407-11411. doi:10.1128/JVI.01211-09

[64] G. Marchetti, P. Nasta, F. Bai, et al., "Circulating sCD14 Is Associated with Virological Response to Pegylated-Interferon-Alpha/Ribavirin Treatment in HIV/HCV Co-Infected Patients," PLoS One, Vol. 7, No. 2, 2012, e32028. www.europeanaidsclinicalsociety.org.

[65] Italian Ministry of Health, "Italian Guidelines for the Use of Antiretroviral Agents and the Diagnostic-Clinical Management of HIV-1 Infected Persons," 2012. http://www.salute.gov.it/hiv/hiv.jsp

[66] G. Cariti, P. Nasta, G. Angarano, et al., "Sustained Virological Response (SVR) to Anti Hepatitis C (HCV) Therapy with Pegylated IFN Alpha (PegIFN) Plus Ribavirin (RBV) in Patient with HIV/HCV Coinfection," ICAR Italian Conference on AIDS and retroviruses, 10-12 June, 2012, Naples, Italy oral communication OC 53.

[67] T. Reiberger, B. A. Payer, L. Kosi, et al. (For the Vienna HIV Coinfection Study Group), "Concomitant Highly
Active Antiretroviral Therapy Leads to Smaller Decline and Faster Recovery of $\mathrm{CD}_{4}+$ Cell Counts During and After Pegylated Interferon on Plus Ribavirin Therapy in HIV-Hepatitis C Virus Coinfected Patients," The Journal of Infectious Diseases, Vol. 203, No. 12, 2011, pp.18021806. doi:10.1093/infdis/jir194

[68] J. Berenguer, M. A. von Wichmann, C. Quereda, et al., "Effect of Accompanying Antiretroviral Drugs on Virological Response to Pegylated Interferon and Ribavirin in Patients Co-Infected with HIV and Hepatitis C Virus," Journal of Antimicrobial Chemotherapy, Vol. 66, No. 12, 2011, pp. 2843-2849. doi:10.1093/jac/dkr362

[69] F. Bani-Sadr, L. Denoeud, P. Morand, et al., "Early Virologic Failure in HIV-Coinfected Hepatitis C Patients Treated with the Peginterferon-Ribavirin Combination: Does Abacavir Play a Role?" Journal of Acquired Immune Deficiency Syndromes, Vol. 45, No. 1, 2007, pp. 123-125. doi:10.1097/QAI.0b013e318040b2b6

[70] C. Solas, E. Pambrun, M. Winnock, et al. (For the ANRS CO-13 HEPAVIH Study Group), "Ribavirin and Abacavir Drug Interaction in HIV-HCV Coinfected Patients: Fact or Fiction? AIDS, Vol. 26, No. 17, 2012, pp. 2193 2199. doi:10.1097/QAD.0b013e32835763a4

[71] P. Nasta, F. Gatti, M. Puoti, et al., "Insulin Resistance Impairs Rapid Virologic Response in HIV/Hepatitis C Virus Coinfected Patients on Peginterferon-alfa-2a," AIDS, Vol. 22, No. 7, 2008, pp. 857-861. doi:10.1097/QAD.0b013e3282fbd1c4

[72] P. Cacoub, F. Carrat, P. Bédossa, et al., "Insulin Resistance Impairs Sustained Virological Response Rate to Pegylated Interferon Plus Ribavirin in HIV-Hepatitis C Virus-Coinfected Patients: HOMAVIC-ANRS HC02 Study," Antiviral Therapy, Vol. 14, 2009, pp. 839-845. doi:10.3851/IMP1298

[73] S. Naggie and M. S. Sulkowski, "Management of Patients Coinfected with HCV and HIV: A Close Look at the Role for Direct-Acting Antivirals," Gastroenterology, Vol. 142, No. 6, 2012, pp. 1324-1334. doi:10.1053/j.gastro.2012.02.012

[74] T. Kakuda, L. Leopold, S. Nijs, et al., "Pharmacoki Netic Interaction between Etravirine or Rilpivirine and Telaprevir in Healthy Volunteers: A Randomised, Two-Way Crossover Trial," 13th International Workshop on Clinical Pharmacology of HIV Therapy, 16-18 April, 2012, Barcelona, Abstract O-18.

[75] E. Hulskotte, H. P. Feng, F. Xuan, et al., "Pharma Cokinetic Interaction between the HCV Protease Inhibitor Boceprevir and Ritonavir-Boosted HIV-1 Protease Inhibitors Atazanavir, Lopinavir, and Darunavir," 19th Conference on Retroviruses and Opportunistic Infections, 5-8 March, 2012, Seattle, Abstract 771LB. 